\title{
SECOND-ORDER CONVERGENCE OF A PROJECTION SCHEME FOR THE INCOMPRESSIBLE NAVIER-STOKES EQUATIONS WITH BOUNDARIES*
}

\author{
THOMAS Y. HOU ${ }^{\dagger}$ AND BRIAN T. R. WETTON ${ }^{\ddagger}$
}

\begin{abstract}
A rigorous convergence result is given for a projection scheme for the Navier-Stokes equations in the presence of boundaries. The numerical scheme is based on a finite-difference approximation, and the pressure is chosen so that the computed velocity satisfies a discrete divergence-free condition. This choice for the pressure and the particular way that the discrete divergence is calculated near the boundary permit the error in the pressure to be controlled and the second-order convergence in the velocity and the pressure to the exact solution to be shown. Some simplifications in the calculation of the pressure in the case without boundaries are also discussed.
\end{abstract}

Key words. projection method, energy estimates

AMS subject classifications. primary 65N10; secondary $76 \mathrm{D} 05$

1. Introduction. In this paper a numerical method for approximating the solutions of the incompressible Navier-Stokes equations in primitive variables (velocity and pressure) is considered. The main difficulty with these variables is how to update the pressure. There is no time evolution equation for the pressure, and the boundary conditions are not given a priori. Much work concerning this question has been published. We refer the reader to the excellent review article by Gresho and Sani [7] and the book by Peyret and Taylor [13]. One interpretation of the pressure is that it acts to project the velocity field onto the space of divergence-free fields. This interpretation leads to a projection method proposed by Chorin [3]. The first convergence result for the projection method in the absence of boundaries was given by Chorin [4]. His analysis also extends to the case in which boundaries are present, but with lower-order convergence. Bell, Colella, and Glaz [2] generalized Chorin's idea to a fully second-order projection method (i.e., second order in space and time) with upwinding differencing. This gives a computationally more robust algorithm. The purpose of this paper is to analyze a second-order projection method, which is a variant of the one used in [2], in the presence of boundaries.

The key is converting the divergence-free condition into a numerical equation for the pressure. Theoretically speaking, one can derive an equation for the pressure, but this equation is almost useless for computational purposes since we cannot evaluate the pressure boundary conditions. However, at the discrete level one can also derive an equation for the pressure by imposing the discrete divergence-free condition. The difficulty in determining the pressure boundary condition can be avoided by using the marker-and-cell (MAC) grid proposed by Harlow and Welsh [8]. Moreover, we can show that the discrete pressure equation is actually a second-order approximation to the analytic pressure equation, including the boundary values. The discrete divergence free condition is also satisfied up to second-order accuracy. These facts allow us to

* Received by the editors April 29, 1991; accepted for publication (in revised form) June 2, 1992. This research was supported in part by Air Force Office of Scientific Research grant AFOSR-90-0090.

$\dagger$ Courant Institute of Mathematical Sciences, 251 Mercer Street, New York, New York, 10012. The work of this author was supported by National Science Foundation grant DMS-9003202 and by a Sloan foundation fellowship.

¥ Department of Mathematics, University of British Columbia, Vancouver, British Columbia V6T 1Y4, Canada. The work of this author was supported by Natural Sciences and Engineering Research Council grant OGP0122105. 
prove second-order convergence in both velocity and pressure.

The convergence analysis relies on discrete energy estimates. To prove nonlinear stability and consistency, we use an asymptotic error expansion technique due to Strang [15]. The computational velocity is compared to an asymptotic expansion that satisfies the discrete equations for the velocity to a high degree of accuracy. The asymptotic solution for the pressure is chosen so that the comparison velocity satisfies the discrete divergence-free condition to high-order accuracy. This approach, which is similar to that used by Chorin in [4], allows us to approximately cancel the error in the pressure. Because of the staggered grid we use, the tangential velocity component does not lie on the boundary and so an approximate velocity boundary condition must be introduced. We show that the boundary condition we use, which is of the reflection type, is stable and second-order accurate. Our convergence result includes the case of nonhomogeneous velocity boundary conditions.

In the time discrete case the discrete equation for the pressure cannot be solved directly: we use an iteration scheme similar to that used in [2] and [3] to find the solution. In the case without boundaries, however, we can simplify the equation for the pressure so that it can be solved directly.

It is interesting to note that in the case without boundaries a variant of the timesplit projection method that Chorin introduced can be shown to be equivalent to the one we use here. In the time-split method the velocity is updated in one time step by completely ignoring the pressure term and the divergence-free condition. Then the intermediate velocity is projected onto a divergence-free field, which gives the value of velocity at the next time step. Although this formulation is quite different from our nonsplit velocity-pressure formulation, we show that these two methods are exactly the same.

The organization of the paper is as follows: $\S 2$ contains a discussion of the method in the case with boundaries, including a description of the grid used, the semidiscrete equations, and their convergence, and the fully discrete equations and their implementation are described. Section 3 describes simplifications to the method for the case in which there are no boundaries. Preliminary computations that verify the convergence results are given in $\S 4$. The technical details involved in the construction of the asymptotic solutions are included in the appendix.

2. Periodic channel (with boundary). To simplify the analysis and to test numerical calculations we consider the two-dimensional periodic channel shown in Fig. 1 as a model domain with boundary. The incompressible Navier-Stokes equations are

$$
\begin{gathered}
\mathbf{u}_{t}=-\mathbf{u} \cdot \nabla \mathbf{u}+\nu \Delta \mathbf{u}-\nabla p \\
\nabla \cdot \mathbf{u}=0
\end{gathered}
$$

where $\mathbf{u}=(u, v)$ are the velocities, $p$ is the pressure, and $\nu$ is the kinematic viscosity. Boundary values for $u$ and $v$ are given on the upper and lower walls, and it is assumed that both $\mathbf{u}$ and $p$ are 1-periodic in the $x$-direction. The condition

$$
\int p=0
$$

fixes the choice of the arbitrary constant in the pressure. Initial data $\mathbf{u}_{0}=\left(u_{0}, v_{0}\right)$ are given, and it is assumed that $\nabla \cdot \mathbf{u}_{0}=0$. By taking the divergence of (1) we obtain

$$
\Delta p=\nabla \cdot(-\mathbf{u} \cdot \nabla \mathbf{u}+\nu \Delta \mathbf{u}) .
$$




$$
\mathrm{u}=\mathrm{u}^{\mathrm{b}, 1}, \mathrm{v}=\mathrm{v}^{\mathrm{b}, 1}
$$

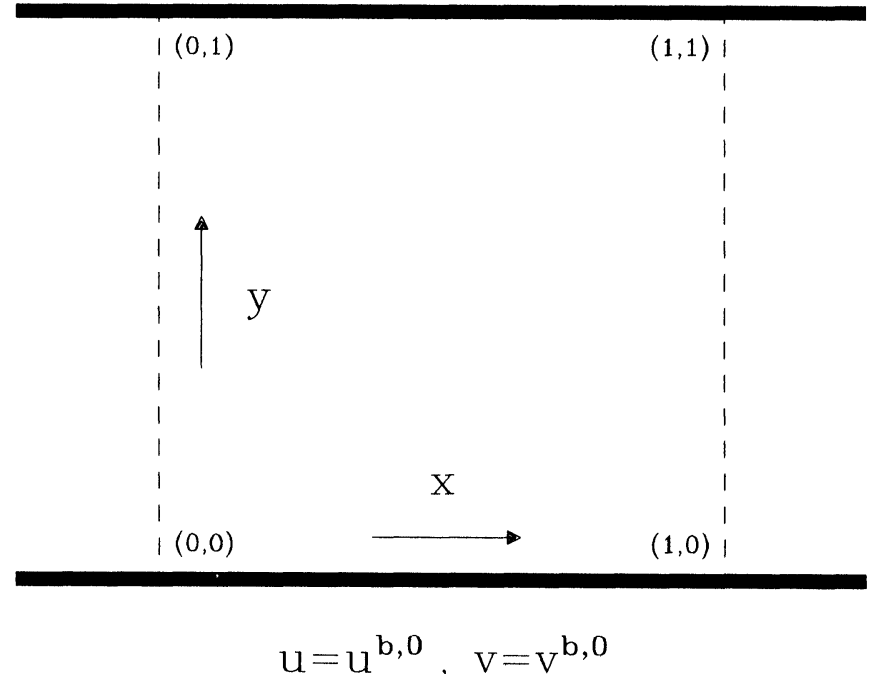

FIG. 1. Periodic channel.

Although the term $\nabla \cdot \Delta \mathbf{u}$ vanishes in the continuous case, it is retained for comparison with the discrete case, in which it has a nonzero contribution from the boundary. By taking the normal $(n)$ component of $(1)$ on the boundary (assuming that the boundary values of $\mathbf{u}$ are time independent) we obtain

$$
\frac{\partial p}{\partial n}=n \cdot(-\mathbf{u} \cdot \nabla \mathbf{u}+\nu \Delta \mathbf{u})
$$

Equation (4) together with boundary conditions (5) form a solvable Neumann problem for the pressure that ensures that the velocity evolves in a divergence-free manner. Note that no boundary conditions for the pressure need be given a priori. The pressure is determined uniquely if the additional constraint (3) is applied. The pressure is chosen exactly so that the right-hand side of (1) is projected orthogonally (in the sense of $L_{2}$ functions) onto the space of divergence-free fields that satisfy homogeneous normal boundary conditions (see Chorin and Marsden [5]).

We propose a semidiscrete scheme below and demonstrate its second-order (in space) convergence. A time-discrete version that is second order in both space and time is then considered. Convergence is obtained in the $l_{2}$ (energy) and maximum norms. Important points in the analysis are that the calculated pressure satisfies (4) and boundary conditions (5) to second-order accuracy and that the discrete divergence condition on the velocities is satisfied. To handle the nonlinear error terms and the coupling between the velocity and pressure errors, we introduce in the manner of Strang [15] an asymptotic solution that satisfies the equations to a high order of accuracy. Although the analysis is performed for the two-dimensional case for simplicity, the results extend to the three-dimensional case, and notes on how to modify the proofs are given. A point of additional interest is that the algorithm gives second-order convergence in the pressure. The simplifications to the algorithm for domains without boundaries are given in $\S 3$. 


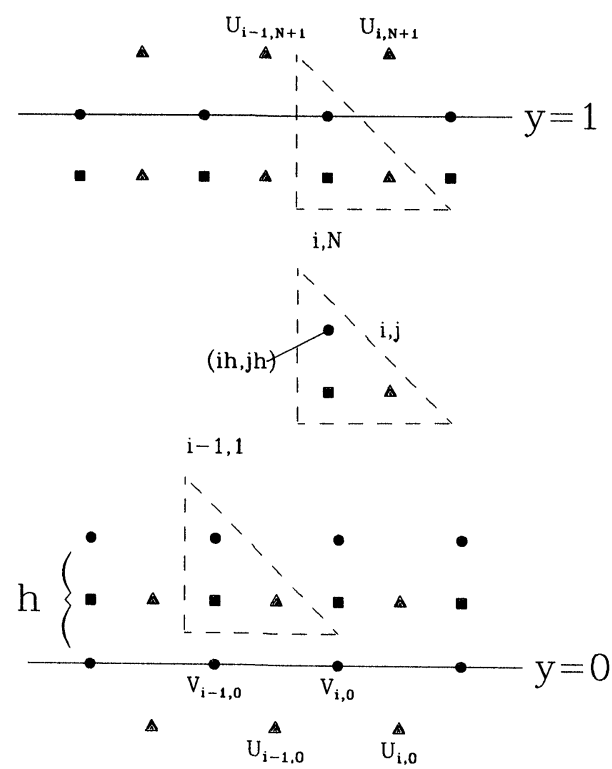

FIG. 2. Discrete grid: the pressure $P$ is defined on square points, the horizontal velocity $U$ at triangles, and the vertical velocity $V$ at circles.

2.1. Semidiscrete equations. We use the MAC staggered grid [8] in the approximation of the velocities and the pressure. That is, we consider approximations

$$
\mathbf{U}_{i, j}(t)=\left(U_{i, j}(t), V_{i, j}(t)\right) \quad \text { and } \quad P_{i, j}(t)
$$

to

$$
u\left(\left(i+\frac{1}{2}\right) h,\left(j-\frac{1}{2}\right) h, t\right), \quad v(i h, j h, t), \quad \text { and } p\left(i h,\left(j-\frac{1}{2}\right) h, t\right),
$$

where $h$ is the grid spacing given by $h=1 / N$. This situation is shown pictorially in Fig. 2. Just as in the analytic case, no boundary values for the pressure need to be specified: the pressure is determined entirely by the divergence-free condition on the velocity. We introduce the difference operators

$$
\begin{array}{rlr}
D_{0}^{x} f_{i, j} & =\frac{f_{i+1, j}-f_{i-1, j}}{2 h} & \text { (centered) } \\
D_{-}^{x} f_{i, j} & =\frac{f_{i, j}-f_{i-1, j}}{h} & (\text { backward) } \\
D_{+}^{x} f_{i, j} & =\frac{f_{i+1, j}-f_{i, j}}{h} & \text { (forward). }
\end{array}
$$

The operators $D_{0}^{y}, D_{-}^{y}$, and $D_{+}^{y}$ are defined similarly. The centered difference approximation to the Laplacian is denoted by $\Delta_{h}$, which can be written as follows:

$$
\Delta_{h}=D_{-}^{x} D_{+}^{x}+D_{-}^{y} D_{+}^{y} .
$$

The discrete divergence of a vector field $\mathbf{U}=(U, V)$ is defined as

$$
\mathbf{D} \cdot \mathbf{U}_{i, j}=D_{-}^{x} U_{i, j}+D_{-}^{y} V_{i, j},
$$


and the discrete gradient of a scalar field $P$ is given by

$$
\mathbf{G} P_{i, j}=\left(D_{+}^{x} P_{i, j}, D_{+}^{y} P_{i, j}\right) .
$$

Note that although $\mathbf{D} \cdot \mathbf{U}$ is given in (12) by using one-sided notation, differences are centered on the scalar pressure points and the differences in $\mathbf{G} P$ are centered at the vector velocity points. Also note that no boundary values for $P$ are needed for obtaining the discrete gradient at interior points.

Let $(\cdot, \cdot)$ be the $l_{2}$ inner product defined on scalar fields (i.e., fields defined on the pressure points):

$$
(P, Q)=h^{2} \sum_{i, j=1}^{N} P_{i, j} Q_{i, j} .
$$

The corresponding norm is denoted by $\|\cdot\|_{2}$. Similar norms and inner products can be defined for vector fields by using the same notation. We restrict our attention first to the case of homogeneous boundary data $u=v=0$. The computational boundary values are given by

$$
\begin{gathered}
V_{i, 0}=V_{i, N}=0, \\
U_{i, 0}=-U_{i, 1}, \\
U_{i, N+1}=-U_{i, N} .
\end{gathered}
$$

Equation (15) is the exact boundary condition, and (16)-(17) are called reflection boundary conditions. If (16)-(17) are interpreted as Taylor series about the boundary, they are satisfied up to second order. In the book by Peyret and Taylor [13] and the article by Gresho and Sani [7] concern is expressed over the use of these reflection boundary conditions in the diffusion terms. Our analysis will show that this concern is not justified. Because the reflection boundary conditions are second-order accurate to the physical no-slip conditions, they will only introduce second-order errors into the computation.

Following Anderson [1], we define the reduced divergence operator $\mathbf{D}^{*}$. as follows: it equals $\mathbf{D}$. at scalar points with $2 \leq j \leq N-1$ and at $j=1$ it is given by

$$
\mathbf{D}^{*} \cdot \mathbf{U}_{i, 1}=D_{-}^{x} U_{i, 1}+\frac{V_{i, 1}}{h}
$$

and at $j=N$ it is given by

$$
\mathbf{D}^{*} \cdot \mathbf{U}_{i, N}=D_{-}^{x} U_{i, N}-V_{i, N-1} / h .
$$

Note that $\mathbf{D}^{*}$. involves only interior variables. Also note that for vector fields with homogeneous boundary values $(15), \mathbf{D}^{*} \cdot \mathbf{U}=\mathbf{D} \cdot \mathbf{U}$. We make two more observations. First, we define

$$
\Delta_{h}^{0} P:=\mathbf{D}^{*} \cdot \mathbf{G} P .
$$

The following result is obtained by using summation-by-parts formulas:

$$
(\mathbf{U}, \mathbf{G} P)=\left(\mathbf{D}^{*} \cdot \mathbf{U}, P\right)
$$

for all scalar fields $P$ and vector fields $U$. This implies that the space of gradient fields and the space of divergence-free fields are orthogonal, just as in the continuous 
case [5]. The following completeness result is obtained by using elementary linear algebra: every discrete periodic vector field $\mathbf{U}$ can be decomposed into the sum of a gradient field $\mathbf{G} P$ and a divergence-free field $\mathbf{U}^{f}$ that satisfies (15). The field $\mathbf{U}^{f}$ is determined uniquely, and $P$ is determined up to a constant. These properties can be seen explicitly from the equation that $P$ must satisfy:

$$
\Delta_{h}^{0} P_{i, j}=\mathbf{D}^{*} \cdot \mathbf{U}_{i, j}
$$

The matrix $\Delta_{h}^{0}$ is symmetric and has a null space consisting of the constant vectors. Since the right-hand side of (22) is orthogonal to constant vectors, (22) is solvable. The choice of the arbitrary constant in $P$ is fixed by requiring that $\sum_{i, j=1}^{N} P_{i, j}=0$. The orthogonal projection $\mathcal{P}$ onto the space of discrete vector fields $\mathbf{U}$ that satisfy $\mathbf{D}^{*} \cdot \mathbf{U}=0$ is defined by

$$
\mathcal{P} \mathbf{U}=\mathbf{U}^{f} .
$$

We are now in a position to describe the semidiscrete numerical method. Let $\tilde{\mathbf{U}}_{i, j}=$ $\left(u_{0}\left(\left(i+\frac{1}{2}\right) h, j h\right), v_{0}\left(i h,\left(j+\frac{1}{2}\right) h\right)\right)$. As initial data for the algorithm, take $\mathbf{U}_{0}=\mathcal{P} \tilde{\mathbf{U}}$. The equation for the velocities is

$$
\dot{\mathbf{U}}=\mathcal{P}\left(-N(\mathbf{U})+\nu \Delta_{h} \mathbf{U}\right),
$$

where $N(\mathbf{U})$ represents the nonlinear convection terms whose discretization will be explained later. Equation (24) is a vector equation for the interior velocities. Since $\mathbf{U}$ starts out divergence free and (24) ensures that it evolves in a divergence-free manner, we have $\mathbf{D} \cdot \mathbf{U}=0$ for all $t$. Equation (24) is equivalent to the pair of equations

$$
\begin{gathered}
\dot{\mathbf{U}}=-N(\mathbf{U})+\nu \Delta_{h} \mathbf{U}-\mathbf{G} P, \\
\Delta_{h}^{0} P=\mathbf{D}^{*} \cdot\left(-N(\mathbf{U})+\nu \Delta_{h} \mathbf{U}\right) .
\end{gathered}
$$

Like (22), equation (26) is uniquely solvable with the side condition

$$
\sum_{i, j=1}^{N} P_{i, j}=0 .
$$

By formally adding in the missing stencil at the boundary, (26) is equivalent to the following equation:

$$
\Delta_{h} P=\mathbf{D} \cdot\left(-N(\mathbf{U})+\nu \Delta_{h} \mathbf{U}\right)
$$

along with boundary conditions

$$
D_{-}^{y} P_{i, 1}=\left(-N(\mathbf{U})+\nu \Delta_{h} \mathbf{U}\right)_{i, 0}^{2}
$$

and

$$
D_{-}^{y} P_{i, N+1}=\left(-N(\mathbf{U})+\nu \Delta_{h} \mathbf{U}\right)_{i, N}^{2} .
$$

The superscript 2 denotes the second component of the vector. Although the boundary values of $-N(\mathbf{U})+\nu \Delta_{h} \mathbf{U}$ are never actually computed (our grid does not have the necessary points), (28) is a second-order approximation of (4) and (29)-(30) are second-order approximations to (5) since the pressure differences are centered at the 
boundary. Thus the proposed algorithm is at least formally second order. This result fits into the general framework espoused by Gresho and Sani [7]-that if the discrete divergence-free condition is applied in the right way, the computed pressure will approximately satisfy the Poisson equation (4) with boundary conditions (5). by

It remains to describe the discretization for $N(\mathbf{U})$. The first component is given

$$
U_{i, j} \cdot D_{0}^{x} U_{i, j}+V_{i, j}^{*} \cdot D_{0}^{y} U_{i, j}
$$

where $V_{i, j}^{*}$ is the average of the four $V$ velocities around the point where $U_{i, j}$ is defined, i.e.,

$$
V_{i, j}^{*}=\frac{1}{4}\left(V_{i, j}+V_{i+1, j}+V_{i, j-1}+V_{i+1, j-1}\right) .
$$

The second component of $N(\mathbf{U})$ is constructed in a similar way. At this point we have completely specified the semidiscrete algorithm. The formal accuracy of the method can be summarized as follows: the initial conditions have been perturbed by $O\left(h^{2}\right)$, the divergence-free condition is satisfied to second order, and the equations and boundary conditions for the velocity and the pressure are second-order accurate. The question of stability and the techniques needed to show convergence are discussed in the next section.

2.2. Convergence of the semidiscrete solution. In this subsection the proof of the convergence of the semidiscrete algorithm described above is given. As in the convergence analysis in [10], an important element is the construction of approximate solutions that satisfy the discrete equations to a high order of accuracy.

LEMma 2.1 (Consistency). Assume that the solution of the Navier-Stokes equations is sufficiently smooth. There exist error expansions $\hat{\mathbf{u}}$ and $\hat{p}$, periodic in $x$, that are $O\left(h^{2}\right)$ perturbations of $\mathbf{u}$ and $p$ (the exact solutions):

$$
\begin{aligned}
\hat{\mathbf{u}}(x, y, t ; h) & =\mathbf{u}(x, y, t)+\sum_{r=1}^{3} h^{2 r} \mathbf{u}^{(r)}(x, y, t), \\
\hat{p}(x, y, t ; h) & =p(x, y, t)+\sum_{r=1}^{3} h^{2 r} p^{(r)}(x, y, t),
\end{aligned}
$$

where the functions $\mathbf{u}^{(r)}$ and $p^{(r)}$ are smooth and their derivatives can be bounded in terms of $\mathbf{u}$ and its derivatives. These functions satisfy the discrete equations to a high order of accuracy in the following sense:

$$
\begin{gathered}
\hat{\mathbf{u}}(0)=\mathbf{U}_{0, i j}+O\left(h^{8}\right), \\
\hat{\mathbf{u}}_{t}=-N(\hat{\mathbf{u}})-\mathbf{G} \hat{p}+\nu \Delta_{h} \hat{\mathbf{u}}+O\left(h^{8}\right), \\
\Delta_{h} \hat{p}=\mathbf{D} \cdot\left(-N(\hat{\mathbf{u}})+\nu \Delta_{h} \hat{\mathbf{u}}\right)+O\left(h^{8}\right), \\
D_{-}^{y} \hat{p}_{i, 1}=\left(-N(\hat{\mathbf{u}})+\nu \Delta_{h} \hat{\mathbf{u}}\right)_{i, 0}^{2}+O\left(h^{8}\right), \\
D_{-}^{y} \hat{p}_{i, N+1}=\left(-N(\hat{\mathbf{u}})+\nu \Delta_{h} \hat{\mathbf{u}}\right)_{i, N}^{2}+O\left(h^{8}\right), \\
\hat{u}\left(x,-\frac{h}{2}\right)=-\hat{u}\left(x,+\frac{h}{2}\right)+O\left(h^{8}\right), \\
\hat{u}\left(x, 1+\frac{h}{2}\right)=-\hat{u}\left(x, 1-\frac{h}{2}\right)+O\left(h^{8}\right),
\end{gathered}
$$




$$
\begin{aligned}
& \hat{v}(x, 0)=\hat{v}(x, 1)=0 \\
& h^{2} \sum_{i, j=1}^{N} \hat{p}_{i, j}=O\left(h^{8}\right)
\end{aligned}
$$

where the terms $O\left(h^{8}\right)$ are smooth.

The terms in (36)-(41) that are outside the domain are given by the Taylor series of these expressions at the boundary to sufficiently high accuracy. A more detailed discussion and the proof of the consistency lemma, which is quite technical, is left to the appendix.

The major convergence result is given below.

THEOREM 2.2 (Convergence). Under the same assumptions as those given in Lemma 2.1, the solutions of the discrete equations (25) and (26) with initial conditions $\mathrm{U}_{0}$ converge in the discrete maximum norm, $\|\cdot\|_{\infty}$, to the exact solutions of the Navier-Stokes equations with second-order accuracy:

$$
\|\mathbf{u}-\mathbf{U}\|_{\infty} \leq C h^{2}
$$

and

$$
\|p-P\|_{\infty} \leq C h^{2}
$$

for all $t$ with $0 \leq t \leq T$, where $C$ depends only on $T$ and the exact solution $\mathbf{u}$ and its derivatives.

Some properties of the equations will be derived before the proof of the theorem is given. The following error terms are defined:

$$
\begin{gathered}
e_{i, j}=\left(e_{i, j}^{1}, e_{i, j}^{2}\right)=\hat{\mathbf{u}}_{i, j}-\mathbf{U}_{i, j}, \\
E_{i, j}=\hat{p}_{i, j}-P_{i, j} .
\end{gathered}
$$

LEMMA 2.3. The pressure error obeys the following equation:

$$
\Delta_{h}^{0} E=\mathbf{D}^{*} \cdot\left(N(\hat{\mathbf{u}})-N(\mathbf{U})+\nu \Delta_{h} e\right)+O\left(h^{8}\right) .
$$

Proof. Use (38) and (39) to remove the boundary stencils on both sides of (37), and obtain

$$
\Delta_{h}^{0} \hat{p}=\mathbf{D}^{*} \cdot\left(N(\hat{\mathbf{u}})+\nu \Delta_{h} \hat{\mathbf{u}}\right)+O\left(h^{8}\right) .
$$

Subtracting (49) from (26) gives the desired result (48).

LEMMA 2.4. $\left\|\mathrm{D}^{*} \cdot e\right\|_{2} \leq O\left(h^{8}\right)$.

Proof. Taking the reduced discrete divergence of (36) using (49), we obtain

$$
\mathbf{D}^{*} \cdot \hat{\mathbf{u}}_{t}=O\left(h^{8}\right) \text {. }
$$

Since $\mathbf{D}^{*} \cdot \mathbf{U}_{0}=0$ by construction, $\mathbf{D}^{*} \cdot \hat{\mathbf{u}}(0)=O\left(h^{8}\right)$ from (35). The above equation can be integrated to give $\mathbf{D}^{*} \cdot \hat{\mathbf{u}}=O\left(h^{8}\right)$. Since the discrete equations are chosen so that $\mathbf{D}^{*} \cdot \mathbf{U}=0$, the result follows.

LEMMA 2.5 (Discrete Poincaré Inequality). The pressure error $E$ satisfies

$$
\|E\|_{2} \leq\|\mathbf{G} E\|_{2}+O\left(h^{8}\right)
$$


Proof. To prove this lemma we note that (27) and (43) imply that $h^{2} \sum_{i, j=1}^{N} E_{i, j}=$ $O\left(h^{8}\right)$. Then Lemma 2.5 becomes a standard exercise.

LEMMA 2.6 (Pressure Stability). Given $T>0$, suppose that $\|e\|_{2} \leq h^{2}$ for $0 \leq t \leq T$. Then there is an increasing function $B(T)$ that depends only on the exact solution $\mathbf{u}$ such that for all $0 \leq t \leq T$

$$
\|E\|_{2} \leq B\left(h^{8}+\frac{\|e\|_{2}}{h^{2}}\right) .
$$

Proof. Taking the inner product of the pressure error equation in Lemma 2.4 with $E$ and summing by parts using (21), we obtain

$$
\|\mathbf{G} E\|_{2}^{2} \leq\|\mathbf{G} E\|_{2} \cdot\|N(\hat{\mathbf{u}})-N(\mathbf{U})\|_{2}+\nu\|\mathbf{G} E\|_{2} \cdot\left\|\Delta_{h} e\right\|_{2}+O\left(h^{8}\right)\|E\|_{2} .
$$

We use Lemma 2.5 on the right-hand side and obtain

$$
\|\mathbf{G} E\|_{2}^{2} \leq\|\mathbf{G} E\|_{2}\left\{\|N(\hat{\mathbf{u}})-N(\mathbf{U})\|_{2}+\nu\left\|\Delta_{h} e\right\|_{2}+O\left(h^{8}\right)\right\}+O\left(h^{16}\right) .
$$

If $\|\mathbf{G} E\|_{2}>h^{8}$, then we can divide the equation by $\|\mathbf{G} E\|_{2}$ and obtain

$$
\|\mathbf{G} E\|_{2} \leq\|N(\hat{\mathbf{u}})-N(\mathbf{U})\|_{2}+\nu\left\|\Delta_{h} e\right\|_{2}+O\left(h^{8}\right) .
$$

Notice that (55) is true if $\|\mathbf{G} E\|_{2} \leq h^{8}$, and so it is always valid. We now linearize the term $N(\hat{\mathbf{u}})-N(\mathbf{U})$ about the solution $\hat{\mathbf{u}}$. Since all the terms are handled in the same way, we consider only the term

$$
\hat{u} \cdot D_{0}^{x} \hat{u}_{i, j}-U_{i, j} \cdot D_{0}^{x} U_{i, j}=\left(e_{i, j}^{1}\right) D_{0}^{x} \hat{u}_{i, j}+\hat{u}_{i, j} \cdot D_{0}^{x} e_{i, j}^{1}+e_{i, j}^{1} \cdot D_{0}^{x} e_{i, j}^{1} .
$$

The first two terms on the right-hand side are bounded by $K\|e\|_{2}$ and $K\|e\|_{2} / h$, respectively, where $K$ depends only on $\hat{v}$ and so on $\mathbf{u}$. To bound the final term on the right we use the embedding

$$
\|f\|_{\infty} \leq \frac{1}{h}\|f\|_{2}
$$

which is true for all discrete functions $f$ (for a proof see [10]). This fact and the assumption that $\|e\|_{2} \leq h^{2}$ gives $\left\|D_{0}^{x} e^{1}\right\|_{\infty} \leq 1$. Combining the above results into the worst term, we obtain

$$
\|N(\hat{\mathbf{u}})-N(\mathbf{U})\|_{2} \leq \frac{B\|e\|_{2}}{h} .
$$

The second term on the right-hand side of (55) is actually the largest in magnitude. We use the brute-force bound

$$
\left\|\Delta_{h} e\right\|_{2} \leq 8 h^{-2}\|e\|_{2}
$$

on the second term. This, together with the bound on the first term (58), is used in (55). After an application of Lemma 2.5 we obtain (52), the desired result.

LEMMA 2.7 (Velocity Stability). Under the same assumptions as in Lemma 2.6 the function $B$ can be chosen so that for all $0 \leq t \leq T$

$$
\frac{d}{d t}\|e\|_{2}^{2} \leq B\left(h^{16}+h^{6}\|e\|_{2}+\|e\|_{2}^{2}\right)
$$


Proof. The evolution equation for the velocity error is

$$
\dot{e}=-(N(\hat{\mathbf{u}})-N(\mathbf{U}))-\mathbf{G} E+\nu \Delta_{h} e+O\left(h^{8}\right) .
$$

After taking the inner product of (61) with $e$, we obtain

$$
\begin{aligned}
\frac{1}{2} \frac{d}{d t}\|e\|_{2}^{2}= & -(e, N(\hat{\mathbf{u}})-N(\mathbf{U})) \\
& -(e, \mathbf{G} E) \\
& +\nu\left(e, \Delta_{h} e\right) \\
& +\left(e, O\left(h^{8}\right)\right) .
\end{aligned}
$$

The terms on the right-hand side above are denoted as convective (62), pressure (63), diffusive (64), and consistency error terms (65). The consistency terms (65) are easily bounded by the second factor in (60), although other factors that can be described as consistency errors will arise in the other terms. These other terms are handled separately below.

Pressure terms. After summation by parts, term (63) becomes

$$
\left(\mathbf{D}^{*} \cdot e, E\right) \text {. }
$$

Lemma 2.4 can be combined with Lemma 2.6 to show that term (63) is bounded by

$$
O\left(h^{6}\right)\|e\|_{2}+O\left(h^{16}\right) .
$$

This technique is similar to that used by Chorin in [4]. The discrete solution is being compared to an expansion that is chosen to reduce the influence of the pressure error term.

Diffusive terms. These terms could be handled easily except for the boundary conditions (16) and (17). It will be shown below that these boundary conditions are stable with respect to the diffusive terms. The boundary conditions (16)-(17) and (40)-(41) for the asymptotic solution can be summarized as

$$
\begin{aligned}
D_{-}^{y} e_{i, 1} & =\frac{2 e_{i, 1}}{h}+O\left(h^{7}\right), \\
D_{-}^{y} e_{i, N+1} & =-\frac{2 e_{i, N}}{h}+O\left(h^{7}\right),
\end{aligned}
$$

where here and in what follows $e$ represents the first component of the velocity error, not the vector error. The term in (64) that cannot be handled in a standard way is

$$
\left(e, D_{+}^{y} D_{-}^{y} e\right)=h^{2} \sum_{i, j=1}^{N} e_{i, j} D_{+}^{y} D_{-}^{y} e_{i, j} .
$$

After summation by parts this becomes

$$
-h^{2} \sum_{i=1}^{N}\left(\sum_{j=2}^{N}\left(D_{-}^{y} e_{i, j}\right)^{2}+\frac{e_{i, 1} D_{-}^{y} e_{i, 1}}{h}-\frac{e_{i, N} D_{-}^{y} e_{i, N+1}}{h}\right),
$$

which after use of (68) and (69) can be written

$$
-h^{2} \sum_{i=1}^{N}\left(\sum_{j=2}^{N}\left(D_{-}^{y} e_{i, j}\right)^{2}+2\left(\frac{e_{i, 1}}{h}\right)^{2}+2\left(\frac{e_{i, N}}{h}\right)^{2}+\left(e_{i, 1}+e_{i, N}\right) \cdot O\left(h^{6}\right)\right)
$$


The fact that the first three terms in (72) have definite sign means that the above expression is less than or equal to $O\left(h^{6}\right)\|e\|_{2}$. This can be absorbed into the second term on the right-hand side of $(60)$. This analysis also gives the following result: the operator $\Delta_{h}$ on vector fields with boundary conditions (15)-(17) is negative definite. To see this, first note that the operator is symmetric and then use the analysis above (and standard techniques on the other terms) to show that

$$
\left(\mathbf{U}, \Delta_{h} \mathbf{U}\right) \leq 0
$$

with equality only when $\mathbf{U} \equiv 0$. This fact is needed to show that the equation for the pressure in the fully discrete setting is solvable.

Convective terms. Again, the way that (62) is shown to be stable would be standard except for the way the boundary conditions (15) and (16) enter the linearized term:

$$
\left(e, \hat{v}^{*} D_{0}^{y} e\right)=h^{2} \sum_{i, j=1}^{N} e_{i, j} \hat{v}_{i, j}^{*} \cdot D_{0}^{y} e_{i, j},
$$

where, as above, $e$ represents only the first component of the velocity error. To prove that this term is stable we need the following:

$$
\hat{v}_{i, 1}^{*}, \hat{v}_{i, N}^{*}=O(h) .
$$

This is true for the exact solution $v$. Since the asymptotic solution $\hat{v}$ is an $O\left(h^{2}\right)$ perturbation of $v$, the result follows. We return now to the expression (74) and rewrite it as

$$
\frac{h^{2}}{2} \sum_{i, j=1}^{N} \hat{v}_{i, j}^{*} \cdot D_{+}^{y}\left(e_{i, j} e_{i, j-1}\right) .
$$

After summation by parts and the use of (68) and (69) this becomes

$$
\begin{aligned}
-\frac{h^{2}}{2} \sum_{i=1}^{N}\left\{\sum_{j=2}^{N} D_{-}^{y} \hat{v}_{i, j}^{*} \cdot e_{i, j} e_{i, j-1}\right. \\
\left.\quad+\frac{\hat{v}_{i, 1}^{*}}{h} e_{i, 1}\left(-e_{i, 1}+O\left(h^{8}\right)\right)-\frac{\hat{v}_{i, N}^{*}}{h} e_{i, N}\left(-e_{i, N}+O\left(h^{8}\right)\right)\right\} .
\end{aligned}
$$

Using (75) and the fact that $\hat{v}$ is smooth so $D_{-}^{y} \hat{v}^{*}$ is $O(1)$, we can bound (77) by $O(1)\|e\|_{2}^{2}+O\left(h^{8}\right)\|e\|_{2}$. Combining the results from this term and the others, we obtain (60), completing the proof of Lemma 2.7.

We are now in a position to prove Theorem 2.2.

Proof. For a given $h$ define $T^{*}$ by

$$
T^{*}=\inf \left\{t:\|e(t)\|_{2}>h^{2}\right\} .
$$

From (35), $\|e(0)\|_{2}=O\left(h^{8}\right)$, and so $T^{*}>0$ for $h$ sufficiently small. We assume that $T^{*}>T$, and then Lemma 2.7 can be applied. We rewrite the quadratic differential inequality (60) as

$$
\frac{d}{d t}\|e\|_{2} \leq \frac{1}{2} B\left(\frac{h^{16}}{\|e\|_{2}}+h^{6}+\|e\|_{2}\right) .
$$


It will be shown that

$$
\|e(t)\|_{2} \leq\left(3 e^{B t / 2}-2\right) h^{6}
$$

for $t<T$. In what follows we will assume that $\|e(0)\|_{2} \leq h^{6}$. If $\|e(t)\| \leq h^{6}$ for all $t<T$, then (81) is trivially satisfied. For $t$ where $\|e(t)\|>h^{6},(80)$ implies

$$
\frac{d}{d t}\|e\|_{2} \leq \frac{1}{2} B\left(h^{10}+h^{6}+\|e\|_{2}\right) \leq \frac{1}{2} B\left(2 h^{6}+\|e\|_{2}\right) .
$$

The above inequality can be integrated from $t_{0}$, where $\left\|e\left(t_{0}\right)\right\|=h^{6}$, to give

$$
\|e(t)\|_{2} \leq\left(3 e^{B\left(t-t_{0}\right) / 2}-2\right) h^{6}
$$

which implies (81). In summary of the proof so far, if $T^{*}>T$, then (81) holds true up to time $T$. A contradiction argument, such as that used in [6], shows that if $h<2 e^{B T / 4}$, then $T^{*}$ must be greater than $T$. It is here that the fact that $B(T)$ is an increasing function is used. If we use the embedding to the max norm on the expression (81), we have $\|e\|_{\infty}=O\left(h^{5}\right)$, or

$$
\|\mathbf{U}-\hat{\mathbf{u}}\|_{\infty}=O\left(h^{5}\right) .
$$

Since $\hat{\mathbf{u}}$ is just an $O\left(h^{2}\right)$ perturbation of the exact velocities $\mathbf{u}$, the result (44) of the theorem follows. Since $\|E\|_{2} \leq B\left(\|e\|_{2} / h^{2}+h^{8}\right)$ for $t<T,\|E\|_{2}=O\left(h^{3}\right)$ and a similar discussion to that above gives (45). This completes the proof of the theorem.

Dependence on the Reynolds number. In the above proof of Lemmas 2.6 and 2.7 (stability) the function $B$ did not depend explicitly on the viscosity $\nu$, and so the same is true of the constant $C$ in the statement of the Convergence Theorem 2.2. However, $C$ does depend on the exact solution, which can be expected to develop large gradients near the boundary when the viscosity is small. Thus $C$ will have an implicit dependence on the Reynolds number.

2.3. Nonhomogeneous boundary data. The case of specified but nonhomogeneous boundary data can be handled in this formulation. Let us consider first the specification of nonzero tangential velocity, i.e., suppose that $u^{b, 0}(x)$ and $u^{b, 1}(x)$ are the specified boundary velocities on the bottom and top walls of the channel. Then the boundary conditions

$$
\begin{aligned}
U_{i, 0} & =2 u_{i}^{b, 0}-U_{i, 1}, \\
U_{i, N+1} & =2 u_{i}^{b, 1}-U_{i, N}
\end{aligned}
$$

can be used. After simple modifications to the Consistency Lemma 2.1 we can demonstrate second-order convergence of this method also.

The case of nonzero normal velocity at the boundary is a little trickier, and there are restrictions on $h$ in the convergence proof. Suppose that the boundary velocities $v^{b, 0}(x)$ and $v^{b, 1}(x)$ are given on the bottom and top walls of the channel. The following compatibility condition on these boundary velocities is forced by the incompressibility condition:

$$
\int_{0}^{1}\left(v^{b, 0}(x)-v^{b, 1}(x)\right) d x=0
$$


If the initial velocity $\mathbf{U}(0)$ satisfies $\mathbf{D} \cdot \mathbf{U}(0)=0$, then the equations that will guarantee that this continues to be satisfied are just (25)-(26) and the algorithm does not need to be modified. However, (75) can no longer be used, and we need to use the diffusive terms to bound the error coming from the linearized convection terms at the boundary. Stability (and hence convergence) can be obtained if

$$
h \leq \frac{2 \nu}{M}
$$

where $M$ is a bound on the velocities at the boundary. In this case the value of $C$ in Theorem 2.2 will depend explicitly on the viscosity. It is not clear whether this is a limitation of the proof or whether instability will actually be observed if (88) is violated. The authors plan to investigate this question computationally.

The question of how to ensure that $\mathbf{D} \cdot \mathbf{U}(0)=0$ remains to be discussed. By using the methods developed by Anderson [1], we can project the exact initial data onto this discrete space as long as

$$
\sum_{i=1}^{N}\left(V_{i, 0}-V_{i, N}\right)=0,
$$

where the values of $V$ in the sum above are given as boundary values. If we take $V_{i, 0}=v_{i}^{b, 0}$ and $V_{i, N}=v_{i}^{b, 1}$, condition (89) will not necessarily be satisfied. Therefore, we compute $\mu$ by using the formula

$$
\mu:=h \sum_{i=1}^{N}\left(v_{i}^{b, 0}-v_{i}^{b, 0}\right)
$$

and set

$$
\begin{aligned}
V_{i, 0} & =v_{i}^{b, 0}-\frac{\mu}{2} \\
V_{i, N} & =v_{i}^{b, 1}-\frac{\mu}{2}
\end{aligned}
$$

which satisfies (89). Since (90) is a midpoint rule approximation of (87), $\mu=O\left(h^{2}\right)$, and so we have made only a second-order perturbation to the exact boundary conditions. To summarize, we modify the boundary values so that they satisfy (89), and we then project the initial data onto the space that satisfies $\mathbf{D} \cdot \mathbf{U}(0)=0$. The Consistency Lemma 2.1 and the rest of the convergence proof can be modified to show convergence provided (88) is satisfied. This approach can also be extended to time-varying boundary values.

2.4. Fully discrete equations and computational issues. In this section a fully discrete implementation of the scheme described above is given and some computational issues are discussed. The method handles the diffusion terms by using a Crank-Nicholson approximation and the convective and pressure terms in a leapfrog manner. The pressure is again completely determined by the condition that the discrete velocity be divergence free, but the equation for the pressure is not solved directly: instead, we describe an iterative procedure for solving the pressure. The fully discrete method is based on the same spatial grid as in the semidiscrete case, with the addition of time discretization at regular intervals of size $k$ in time. The superscript on discrete variables will denote the time step. Assume that $\mathbf{U}^{0}$ and 
$\mathbf{U}^{1}$ are given approximations to the velocity at time zero and time $k$ that satisfy $\mathbf{D} \cdot \mathbf{U}^{0}=\mathbf{D} \cdot \mathbf{U}^{1}=0$. The discretization for the momentum equations is

$$
\frac{\mathbf{U}^{n+1}-\mathbf{U}^{n-1}}{2 k}=-N\left(\mathbf{U}^{n}\right)-\mathbf{G} P^{n}+\frac{1}{2} \nu \Delta_{h}\left(\mathbf{U}^{n+1}+\mathbf{U}^{n-1}\right) .
$$

In what follows we consider homogeneous boundary conditions for simplicity, although modifications for the general case can be made as described above. If we solve formally for $\mathbf{U}^{n+1}$, we obtain

$$
\mathbf{U}^{n+1}=\left(I-k \nu \Delta_{h}\right)^{-1}\left(-2 k N^{n}-2 k \mathbf{G} P^{n}+\left(I+k \nu \Delta_{h}\right) \mathbf{U}^{n-1}\right),
$$

where $I$ is the identity matrix on vector fields. Simply by applying $\mathbf{D}^{*}$. to (94) and setting the resulting expression to zero (to ensure that the velocity at the next step is divergence free) we obtain the equation for the pressure:

$$
2 k \mathbf{D}^{*} \cdot\left(I-k \nu \Delta_{h}\right)^{-1} G P^{n}=\mathbf{D}^{*} \cdot\left(-2 k N^{n}+\left(I+k \nu \Delta_{h}\right) \mathbf{U}^{n-1}\right) .
$$

Using the symmetry of the vector operator $\Delta_{h}$ and the fact that it is negative definite (see the note after (72)), we can show that this equation has a unique solution for $P^{n}$ with the side condition (27). The following theorem can be proved (see [18]).

THEOREM 2.8 (Convergence). Suppose that $k$ is related to $h$ by the fixed ratio $k=\lambda h$, where $\lambda=\sup |\mathbf{u}| / 2$. Then the approximate solutions $\mathbf{U}$ and $P$ described above converge to the exact values with second-order accuracy in space and time.

To prove convergence we have to be a little careful in the choice of initial data: we can take $\mathbf{U}^{0}=\mathcal{P} \mathbf{u}(0)$ as before, but the analysis requires that $\mathbf{U}^{1}$ match the asymptotic solution $\hat{\mathbf{u}}$ to a high degree of accuracy. Theoretically, this could be done by computing Taylor series of the asymptotic solution at the initial time, but in practice the computation is not necessary. In the computations described in $\S 4.1$ we use a single forward Euler step to approximate $\mathbf{U}^{1}$, and this is sufficient to observe second-order convergence.

Unfortunately, we cannot compute the solution to (95) directly in a computationally efficient way. Equation (93) and $\mathbf{D}^{*} \cdot \mathbf{U}^{n+1}=0$ can be considered as a coupled, definite system to be solved for $\mathbf{U}^{n+1}$ and $P^{n}$ simultaneously. To avoid solving this coupled system we replace (95) by an iterative scheme. We use a second superscript to denote the iteration level. We take $P^{n-1}$ as the initial guess for the value of $P^{n}$, i.e., $P^{n, 0}=P^{n-1}$. Then we compute

$$
\begin{gathered}
\mathbf{U}^{n+1, j+1}=\left(I-k \nu \Delta_{h}\right)^{-1}\left(-2 k N^{n}-2 k \mathbf{G} P^{n, j}+\left(I+k \nu \Delta_{h}\right) \mathbf{U}^{n-1}\right), \\
2 k \mathbf{D}^{*} \cdot \mathbf{G} P^{n, j+1}=\mathbf{D}^{*} \cdot\left(-2 k N^{n}+\left(I+k \nu \Delta_{h}\right) \mathbf{U}^{n-1}+k \nu \Delta_{h} \mathbf{U}^{n, j+1}\right) .
\end{gathered}
$$

The above iteration has a fixed point of (93), (95), as desired. The algorithm described above is similar to that described by Bell, Colella, and Glaz in [2]. These authors use a different approximation to the nonlinear terms $N^{n}$ that is based only on the velocities at time step $n-1$ (so it is not a two-level method) and that has desirable properties for computing flows at high Reynolds numbers. It can be shown, as Bell, Colella, and Glaz claim for their scheme [2], that the above iteration, when considered as a map for the discrete pressure, is a contraction. If this iteration were carried to sufficient accuracy, then the convergence result of Theorem 2.8 would also apply. However, there is a certain difficulty since the contraction parameter for the pressure map tends to 1 as $h \rightarrow 0$, as in the following formula:

$$
\frac{\nu \lambda}{h+\nu \lambda}
$$


where $\lambda$ is described in Theorem 2.8. In the computational example described below, this difficulty does not manifest itself: we observe convergence of the scheme by using a fixed, small number of iterations. The authors plan to examine the convergence properties of this iteration scheme in future work.

2.5. A note on higher dimensions and other extensions. The staggered grid and the numerical scheme described above in two dimensions are easily generalized to three or higher dimensions. The only part of the convergence analysis that is specific to two dimensions is the embedding (57). In $n$ dimensions this becomes

$$
\|f\|_{\infty} \leq h^{-n / 2}\|f\|_{2}
$$

By increasing the number of terms in the expansions (33) and (34) and so increasing the accuracy of the asymptotic solutions, the proof of Theorems 2.2 and 2.8 can be extended to any dimension by using similar simple geometries.

For practical computations, several additional questions must be addressed: the use of inflow and outflow boundary conditions, the performance of the method in domains with corners, and the use of curvilinear grids for more general geometries. The analysis presented in this paper has essentially two assumptions, that the underlying flow is smooth and that the computational method used is linearly stable. The convergence of a method that uses inflow and outflow boundary conditions basically reduces to checking the linear stability of the method by using those boundary conditions. This was considered by Naughton in [12] for several kinds of boundary conditions. The smoothness assumption in the analysis means that our results cannot be applied directly to flows in domains with corners where singularities in the flow can be expected to develop. A projection method can be formulated for such a case (i.e., flow over a step), and it may be possible to observe convergence away from the corner due to smoothing properties of the method. The final extension of interest is to curvilinear grids. The projection scheme based on the MAC grid considered in this paper, which is amenable to analysis, is unfortunately not obviously extensible to curvilinear coordinates. The development and analysis of a projection method for general geometries using other approaches is one of the authors' current research areas.

3. Periodic flow (without boundary). We now consider the incompressible Navier-Stokes equations in a periodic box $\Omega=[0,1] \times[0,1]$. Using the discrete grid discussed in $\S 2.1$, we can formulate a semidiscrete numerical method in the case without boundaries:

$$
\begin{gathered}
\dot{\mathbf{U}}=-N(\mathbf{U})+\nu \Delta_{h} \mathbf{U}-\mathbf{G} P, \\
\Delta_{h} P=\mathbf{D} \cdot\left(-N(\mathbf{U})+\nu \Delta_{h} \mathbf{U}\right) .
\end{gathered}
$$

Since the velocity starts out divergence free and the evolution of the velocity is such that it remains divergence free and the discrete operators $\mathbf{D}$. and $\Delta_{h}$ commute in this case without boundaries, the equation for the pressure reduces to

$$
\Delta_{h} P=-\mathbf{D} \cdot N(\mathbf{U}) .
$$

This equation is uniquely solvable with the side condition (27).

We now turn to a fully discrete algorithm for the periodic case. The method is similar to the one described in the case with boundaries. It is also in the format of Crank-Nicholson in the diffusive terms and leapfrog in the convective and pressure 
terms. Again, the pressure is chosen to ensure that the velocity satisfies a discrete divergence-free condition.

The pressure at time step $n$ is computed by using the formula

$$
\Delta_{h} P^{n}=-\mathbf{D} \cdot N\left(\mathbf{U}^{n}\right) .
$$

Now the velocity at the next time step can be calculated by using the following implicit scheme:

$$
\frac{\mathbf{U}^{n+1}-\mathbf{U}^{n-1}}{2 k}=-N\left(\mathbf{U}^{n}\right)-\mathbf{G} P^{n}+\frac{1}{2} \nu \Delta_{h}\left(\mathbf{U}^{n+1}+\mathbf{U}^{n-1}\right) .
$$

The statement made earlier that this scheme ensures the discrete divergence-free condition at step $n+1$ remains to be proved. Assume that $\mathbf{U}^{n-1}$ is divergence free. Taking the divergence of (104) and using (103) and the fact that D. and $\Delta_{h}$ commute, we obtain

$$
\left(I-k \nu \Delta_{h}\right) \mathbf{D} \cdot \mathbf{U}^{n+1}=0 .
$$

Since the matrix $I-k \nu \Delta_{h}$ is invertible, we have $\mathbf{D} \cdot \mathbf{U}^{n+1}=0$, as desired. The following theorem can be proved (see [18]).

THEOREM 3.1 (Convergence). Suppose that $k$ is related to $h$ by the fixed ratio $k=\lambda h$, where $\lambda=\sup |\mathbf{u}| / 2$. Then the approximate solutions $\mathbf{U}$ and $P$ described above converge to the exact values with second-order accuracy.

One difference in this case without boundaries is that we have a simple equation for the pressure (103) and do not need to iterate to solve it. The authors believe that a corresponding simplification can be made in the computations in [2].

There is a final point of interest to make in this section. We will show the equivalence of the algorithm described above and one that can more properly be called a "projection" method. Suppose we are given velocities $\mathbf{U}^{n-1}$ and some approximation of the nonlinear terms $N^{n}$. The velocity field $\tilde{\mathbf{U}}^{n+1}$ is calculated with the help of an intermediate velocity $\mathbf{U}^{*}$ as follows:

$$
\begin{gathered}
\frac{\mathbf{U}^{*}-\mathbf{U}^{n-1}}{2 k}=-N^{n}+\frac{1}{2} \nu \Delta_{h}\left(\mathbf{U}^{*}+\mathbf{U}^{n-1}\right) \\
\tilde{\mathbf{U}}^{n+1}=\mathcal{P} \mathbf{U}^{*}
\end{gathered}
$$

The above method is a fractional-step method because we forget about the pressure when we compute (106) and then correct the error we introduce by projecting back onto the space of divergence-free fields. The claim is that $\mathbf{U}^{n+1}$ computed by using (103) and (104) above is the same as $\tilde{\mathbf{U}}^{n+1}$. To see that this is true we write (103)(104) in the equivalent form

$$
\frac{\mathbf{U}^{n+1}-\mathbf{U}^{n-1}}{2 k}=\mathcal{P}\left(-N^{n}\right)+\frac{1}{2} \nu \Delta_{h}\left(\mathbf{U}^{n+1}+\mathbf{U}^{n-1}\right) .
$$

By taking the projection $\mathcal{P}$ of (106) we obtain

$$
\frac{\tilde{\mathbf{U}}^{n+1}-\mathbf{U}^{n-1}}{2 k}=\mathcal{P}\left(-N^{n}\right)+\mathcal{P}\left(\frac{1}{2} \nu \Delta_{h}\left(\mathbf{U}^{*}+\mathbf{U}^{n-1}\right)\right),
$$

where the fact that $\mathbf{D} \cdot \mathbf{U}^{n-1}=0$ so $\mathcal{P} \mathbf{U}^{n-1}=\mathbf{U}^{n-1}$ has been used. Clearly the two computed values would be the same as long as we can commute $\mathcal{P}$ with $\Delta_{h}$ in the above formula. This is easily verified by using the fact that $\mathbf{D} \cdot \mathbf{G}$, and $\Delta_{h}$ commute in the periodic case. 
TABLE 1

Errors in velocity $\mathbf{u}$ and pressure $p$ at time 1 for the periodic channel when 1 iteration for the pressure is used. The errors are normalized by the maximum of the exact values and by $h^{2}$ as shown. Times are in seconds on a SPARC workstation.

\begin{tabular}{|c|ccc|}
\hline \multirow{2}{*}{$N=1 / h$} & \multicolumn{3}{|c|}{$L_{\infty}$-error } \\
\cline { 2 - 4 } & $\mathbf{u}$ & $p$ & Time \\
\hline 8 & $2.0 h^{2}$ & $6.0 h^{2}$ & 1.0 \\
16 & $2.1 h^{2}$ & $5.5 h^{2}$ & 8.2 \\
32 & $2.2 h^{2}$ & $5.5 h^{2}$ & 70 \\
64 & $2.1 h^{2}$ & $5.5 h^{2}$ & 586 \\
\hline
\end{tabular}

4. Numerical results. Simple test cases for domains both with and without boundaries are presented below. Second-order convergence is obtained in both cases.

4.1. Periodic channel. The method described in $\S 2.4$ for calculating flows in a channel (with iteration for the pressure) is applied to the following example:

$$
\begin{gathered}
u(x, y, t)=\frac{1}{4} \sin (2 \pi x) \sin (2 \pi y) e^{-8 \nu \pi^{2} t} \\
v(x, y, t)=\frac{1}{4} \cos (2 \pi x)(\cos (2 \pi y)-1) e^{-8 \nu \pi^{2} t},
\end{gathered}
$$

with pressure

$$
\begin{aligned}
p(x, y, t)= & \left(\cos (4 \pi x)-\frac{\cos (4 \pi y)}{64}\right. \\
& \left.-\frac{\cos (4 \pi x) \cos (2 \pi y)}{80}+\frac{\cos (2 \pi y)}{16}\right) e^{-16 \nu \pi^{2} t}
\end{aligned}
$$

These functions do not satisfy the Navier-Stokes equations exactly, and so we introduce a forcing function $f$ so that

$$
\mathbf{u}_{t}=-\mathbf{u} \cdot \nabla \mathbf{u}-\nabla p-\nu \Delta \mathbf{u}+f .
$$

In this case the forcing function $f$ is given by

$$
\begin{aligned}
f(x, y, t)=\left(-\pi \sin (4 \pi x) \cos (2 \pi y) e^{-16 \nu \pi^{2} t} / 40,\right. \\
\\
\left.\quad \pi \cos (4 \pi x) \sin (2 \pi y) e^{-16 \nu \pi^{2} t} / 40+\nu \pi^{2} \cos (2 \pi x) e^{-8 \nu \pi^{2} t}\right) .
\end{aligned}
$$

This function $f$ is divergence free and satisfies the boundary conditions of the velocity (the gradient part has been absorbed by the pressure). Making straightforward modifications to the algorithm, we can handle the forcing function. The velocities at time step 1 are calculated with a single forward Euler step, which also furnishes a pressure that is used as the first value in the iterative scheme that is used in the first full step calculation.

The Reynolds number is chosen as 50 , corresponding to a viscosity of 0.01 , and the values are calculated to time 1 and are then compared to the exact values. The results are given in Tables 1 (for 1 iteration) and 2 (for 3 iterations). The errors are normalized by the maximum of the exact velocity or pressure at the final time. The errors are also normalized by $h^{-2}$ so that if the method converged with second-order accuracy, the numbers should remain bounded. This is clearly observed. The fact that the normalized errors are approximately constant shows that the computations are in the asymptotic region of convergence. 
TABLE 2

Errors in velocity $\mathbf{u}$ and pressure $p$ at time 1 for the periodic channel when 3 iterations for the pressure are used. The errors are normalized by the maximum of the exact values and by $h^{2}$ as shown. Times are in seconds on a SPARC workstation.

\begin{tabular}{|c|ccc|}
\hline \multirow{3}{*}{$N=1 / h 1$} & \multicolumn{3}{|c|}{$L_{\infty}$-error } \\
\cline { 2 - 4 } & $\mathbf{u}$ & $p$ & Time \\
\hline 8 & $2.1 h^{2}$ & $6.2 h^{2}$ & 1.7 \\
16 & $2.1 h^{2}$ & $6.0 h^{2}$ & 14 \\
32 & $2.1 h^{2}$ & $5.8 h^{2}$ & 119 \\
64 & $2.1 h^{2}$ & $5.7 h^{2}$ & 988 \\
\hline
\end{tabular}

TABLE 3

Errors in velocity $\mathbf{u}$ and pressure $p$ at time 1 for the doubly periodic geometry, normalized by the maximum of the exact values and by $h^{2}$ as shown. Times are in seconds on a SPARC workstation.

\begin{tabular}{|c|ccc|}
\hline \multirow{3}{*}{$N=1 / h$} & \multicolumn{3}{|c|}{$L_{\infty}$-error } \\
\cline { 2 - 4 } & $\mathbf{u}$ & $p$ & Time \\
\hline 8 & $2.4 h^{2}$ & $3.7 h^{2}$ & 0.7 \\
16 & $2.4 h^{2}$ & $3.1 h^{2}$ & 4.9 \\
32 & $2.4 h^{2}$ & $2.8 h^{2}$ & 35 \\
64 & $2.4 h^{2}$ & $2.7 h^{2}$ & 274 \\
\hline
\end{tabular}

4.2. Periodic box. The method described in $\S 3$ was used to calculate the following exact flow in a periodic cell:

$$
\begin{aligned}
& u(x, y, t)=\frac{1}{2} \sin (2 \pi x) \cos (2 \pi y) e^{-8 \nu \pi^{2} t} \\
& v(x, y, t)=-\frac{1}{2} \cos (2 \pi x) \sin (2 \pi y) e^{-8 \nu \pi^{2} t}
\end{aligned}
$$

with corresponding pressure

$$
p(x, y, t)=\frac{1}{8}(\cos (4 \pi x)+\sin (4 \pi y)) e^{-16 \nu \pi^{2} t} .
$$

Since the velocities are bounded by $\frac{1}{2}$, the relationship $k=h$ is used. The Reynolds number is chosen as 50, corresponding to a viscosity of 0.01 . The computations were initialized with the exact values and the errors in the calculated velocities and pressures at time 1 are given in Table 3 . The errors are normalized by the maximum of the exact values as before. Second-order convergence is clearly observed.

5. Summary. In the literature there has been much discussion of exactly how to handle the pressure and hence the incompressibility condition in the context of finite-difference schemes. Again, we refer the reader to the excellent review article by Gresho and Sani [7] for a summary of previous work. In the present article we have demonstrated one scheme for which we prove rigorous convergence in the cases with boundaries and without. An important element in the scheme is the particular choice of a staggered grid. Using this grid, we see that by choosing the pressure to make the velocity satisfy a discrete divergence-free condition, we also compute an approximation to the pressure that is second-order accurate. Both of these properties, accuracy in the pressure and the discrete divergence-free condition, are needed to fully analyze the sources of error and show convergence of the scheme. We hope that the analysis and discussion of computational issues presented here will shed some light on the gray areas associated with the handling of the pressure in similar schemes. Numerical work to test the performance of the methods proposed here on more complicated flows is currently in progress. 
Appendix: consistency lemmas. An essential part of the convergence analysis is the construction of the asymptotic solutions (33)-(34) that satisfy the discrete equations to high-order accuracy. In this appendix we derive the form of the equations that the functions $\mathbf{u}^{(r)}$ and $p^{(r)}$ must satisfy and indicate why these equations will have smooth solutions. The question of smoothness up to time $t=0$ and the additional compatibility conditions required for this will be addressed briefly at the end of the section. The results below constitute a proof of Lemma 2.1.

We proceed in the manner of Strang [15] and insert the expressions (33)-(34) into the difference equations (25) and (28) as well as the boundary conditions (15)-(17) and (29), (30). We then expand the finite differences in Taylor series and set the coefficients of powers of $h$ in the resulting expression to zero. What we end up with are equations and boundary conditions for the functions $\mathbf{u}^{(r)}$ and $p^{(r)}$. Since the error expansions of all differences involve only even powers of $h$, we need consider only even powers in the resulting expansion. We obtain the exact Navier-Stokes equations as the zeroth-order term (indicating that the scheme is consistent) and equations of the following form for higher-order terms:

$$
\begin{gathered}
\mathbf{u}_{t}^{(r)}=-\mathbf{u}^{(r)} \cdot \nabla \mathbf{u}-\mathbf{u} \cdot \nabla \mathbf{u}^{(r)}-\nabla p^{(r)}+\nu \Delta \mathbf{u}^{(r)}+f^{(r)} \\
\Delta p^{(r)}=-\nabla \cdot\left(\mathbf{u}^{(r)} \cdot \nabla \mathbf{u}-\mathbf{u} \cdot \nabla \mathbf{u}^{(r)}\right)+g^{(r)}
\end{gathered}
$$

with boundary conditions

$$
\begin{gathered}
v^{(r)}=0, \\
u^{(r)}=b^{(r)}, \\
\frac{\partial p^{(r)}}{\partial n}=n \cdot\left(-\mathbf{u}^{(r)} \cdot \nabla \mathbf{u}-\mathbf{u} \cdot \nabla \mathbf{u}^{(r)}+\nu \Delta \mathbf{u}^{(r)}\right)+c^{(r)}
\end{gathered}
$$

at $y=0$ and $y=1$. In the above equations $f^{(r)}, g^{(r)}, b^{(r)}$, and $c^{(r)}$ involve derivatives of $\mathbf{u}, p, \mathbf{u}^{(q)}$, and $p^{(q)}$ for $q<r$. We consider solving (120) and (121) inductively for $r$ with boundary conditions (122)-(124). These equations have convection terms linearized about the exact solution $\mathbf{u}$ and are forced by lower-order terms.

The first question that we need to address is whether the Neumann boundary conditions for $p^{(r)}$ are compatible with the Poisson data for $p^{(r)}$ in the interior. The compatibility condition is

$$
\begin{aligned}
\int_{\Omega}- & \nabla \cdot\left(\mathbf{u}^{(r)} \cdot \nabla \mathbf{u}-\mathbf{u} \cdot \nabla \mathbf{u}^{(r)}\right)+g^{(r)} \\
& =\int_{\partial \Omega} n \cdot\left(-\mathbf{u}^{(r)} \cdot \nabla \mathbf{u}-\mathbf{u} \cdot \nabla \mathbf{u}^{(r)}+\nu \Delta \mathbf{u}^{(r)}\right)+c^{(r)}
\end{aligned}
$$

The first term on the left-hand side can be intergrated by parts to give the first term on the right-hand side. Therefore, we need only check whether

$$
\int_{\Omega} g^{(r)}=\int_{\partial \Omega} c^{(r)}
$$

For the second-order terms we have

$$
\begin{gathered}
g^{(2)}=-\frac{1}{12}\left(\frac{\partial^{4}}{\partial x^{4}} p+\frac{\partial^{4}}{\partial y^{4}} p\right)+\frac{1}{24}\left(\frac{\partial^{3}}{\partial x^{3}} a_{1}^{(0)}+\frac{\partial^{3}}{\partial y^{3}} a_{2}^{(0)}\right)+\nabla \cdot a^{(2)} \\
c^{(2)}=a_{2}^{(2)}-\frac{1}{24} \frac{\partial^{3}}{\partial y^{3}} p
\end{gathered}
$$


where $a^{(0)}=-\mathbf{u} \cdot \nabla \mathbf{u}+\nu \Delta \mathbf{u}$ and $a^{(2)}$ is the second-order error in the expression $-N(\hat{\mathbf{u}})+\nu \Delta \hat{\mathbf{u}}$ that involves the exact solution $\mathbf{u}$ only, i.e., the second-order error in this expression that is not explicitly given in (120). The $a$ 's are vector functions, and the subscript denotes the component. In terms of the compatibility (127) we can ignore all terms with $x$ derivatives since they integrate to zero (all functions are periodic in this direction). We use the relation $\sim$ between two functions that differ only by terms with $x$ derivatives. Applying this notation to (4), we have

$$
\frac{\partial^{2}}{\partial y^{2}} p \sim \frac{\partial}{\partial y} a_{2}^{(0)}
$$

Using this result in (128) and (129), we obtain

$$
\begin{aligned}
g^{(2)} & \sim-\frac{1}{24} \frac{\partial^{3}}{\partial y^{3}} a_{2}^{(0)}+\frac{\partial}{\partial y} a_{2}^{(2)} \\
c^{(2)} & \sim-\frac{1}{24} \frac{\partial^{2}}{\partial y^{2}} a_{2}^{(0)}+a_{2}^{(2)} .
\end{aligned}
$$

By integrating the left-hand side of (127) by parts using the expressions above, we see that the compatibility condition is indeed satisfied. The compatibility of the higher-order terms can also be shown. The lengthy details are given in [18].

The next point is how to satisfy (43). By considering (27) as a midpoint-rule approximation of (3) and expanding the error in powers of $h$ (see [14]) we obtain values for

$$
\int_{\Omega} p^{(r)}(x, y)
$$

in terms of derivatives of lower-order terms in the error expansion. Since the Neumann problem for $p^{(r)}$ is determined only up to a constant, we can satisfy this condition and so satisfy (43). The third point to consider is the projection of the initial data onto the space of discrete divergence-free fields. We write

$$
\hat{\mathbf{u}}(0)=\mathbf{u}(0)+\sum_{r=1}^{3} h^{2 r} \mathbf{u}^{(r)}(0) .
$$

As the above notation suggests, this error expansion furnishes initial data for (120). To satisfy (35) we introduce an initial pressure with an error expansion and choose it so that it satisfies (22) to high-order accuracy. By applying the Taylor series expansion of the discrete gradient to this pressure, we obtain the terms in the initial expansion (134). Now we need only to address the smoothness of the solutions of (120). We rewrite (120)-(121) as follows:

$$
\mathbf{u}_{t}^{(r)}=\mathcal{P}_{c}\left(-\mathbf{u}^{(r)} \cdot \nabla \mathbf{u}-\mathbf{u} \cdot \nabla \mathbf{u}^{(r)}+\nu \Delta \mathbf{u}^{(r)}\right)+f^{(r)}
$$

where $\mathcal{P}_{c}$ denotes the projection onto continuous divergence-free fields and $f^{(r)}$ contains additional terms from (121). It can be shown that this equation has smooth solutions, and we refer the reader to the relevant literature [11], [16]. With this fact the proof of Lemma 2.1 is complete.

$A$ remark on the smoothness of solutions at time $t=0$. It is well known that the solutions of the Navier-Stokes equations are smooth up to time $t=0$ only under certain assumptions on the initial velocity: it must satisfy certain nonlocal compatibility 
conditions. A discussion of these conditions is given in the article by Temam [17]. In addition, the smoothness of the terms of the asymptotic error expansion up to time $t=0$ requires that the initial velocity satisfy additional compatibility conditions. As Heywood and Rannacher remark in [9], it is impossible in general to verify that given initial data satisfy these compatibility conditions. Using the smoothing properties of the diffusive term, they prove the convergence of finite-element approximations to solutions of the Navier-Stokes equations without assuming that any of the compatibility conditions are satisfied. We believe that this approach can be extended to the analysis here.

Acknowledgment. We thank Professor Chris Anderson for bringing this problem to our attention and for several beneficial discussions. We also thank the reviewers, Christoph Borgers and Luis Reyna, for many insightful comments.

\section{REFERENCES}

[1] C. R. Anderson, Derivation and solution of the discrete pressure equations for the incompressible Navier-Stokes equations, preprint LBL-26353, Lawrence Berkeley Laboratory, Berkeley, CA, 1988.

[2] J. B. Bell, P. Colella, AND H. M. Glaz, A second-order projection method for the incompressible Navier-Stokes equations, J. Comput. Phys., 85 (1989), pp. 257-283.

[3] A. J. Chorin, Numerical solution of the Navier-Stokes equations, Math. Comp., 22 (1968), pp. 745-762.

[4] - On the convergence of discrete approximations to the Navier-Stokes equations, Math. Comp., 23 (1969), pp. 341-353.

[5] A. J. ChORIn AND J. E. MARsden, A Mathematical Introduction to Fluid Mechanics, SpringerVerlag, New York, 1990.

[6] J. Goodman, T. Y. Hou, And J. Lowengrub, Convergence of the point vortex method for the 2-D Euler equations, Comm. Pure Appl. Math., 43 (1990), pp. 415-430.

[7] P. M. GRESHO AND R. L. SANI, On pressure boundary conditions for the incompressible Navier-Stokes equations, Internat. J. Numer. Methods Fluids, 7 (1987), pp. 1111-1145.

[8] F. H. HARLOW AND J. E. WELSH, Numerical calculation of time-dependent viscous incompressible flow of fluid with free surfaces, Phys. Fluids, 8 (1965), pp. 2181-2189.

[9] J. G. HEYWOOD AND R. RANNACHER, Finite element approximation of the non-stationary Navier-Stokes problem. I. Regularity of solutions and second-order error estimates for spatial discretization, SIAM J. Numer. Anal., 19 (1982), pp. 275-311.

[10] T. Y. Hou AND B. T. R. WetTon, Convergence of a finite difference scheme for the NavierStokes equations using vorticity boundary conditions, SIAM J. Numer. Anal., 29 (1992), pp. 615-639.

[11] H.-O. Kreiss AND J. Lorenz, Initial-Boundary Value Problems and the Navier-Stokes Equations, Academic Press, San Diego, CA, 1989.

[12] M. J. NAUghton, On numerical boundary conditions for the Navier-Stokes equations, Ph.D. thesis, Department of Applied Mathematics, California Institute of Technology, Pasadena, CA, 1986.

[13] R. Peyret AND T. D. TAYlor, Computational Methods for Fluid Flow, Springer-Verlag, New York, 1983.

[14] P. A. Raviart, An analysis of particle methods, in Numerical Methods in Fluid Dynamics, F. Brezzi, ed., Lecture Notes in Math., 1127, Springer-Verlag, Berlin, 1985.

[15] G. Strang, Accurate partial differential methods II. Non-linear problems, Numer. Math., 6 (1964), pp. 37-46.

[16] R. Temam, Navier-Stokes Equations, North-Holland, New York, 1977.

[17] - Behavior at time $t=0$ of the solutions of semi-linear evolution equations, J. Differential Equations, 43 (1982), pp. 73-92.

[18] B. T. R. WetTon, Convergence of numerical approximations for the Navier-Stokes equations with boundaries: Vorticity and primitive formulations, Ph.D. thesis, Courant Institute of Mathematical Sciences, New York, 1991. 